\title{
Lightweight and stiff cellular ceramic structures by ice templating
}

\author{
Florian Bouville $^{\mathrm{a}, \mathrm{b}}$, Eric Maire ${ }^{\mathrm{b}}$, Sylvain Deville ${ }^{\mathrm{a}}$ \\ a Laboratoire de Synthèse et Fonctionnalisation des Céramiques, UMR3080 \\ CNRS/Saint-Gobain, Cavaillon, France \\ b Université de Lyon, INSA-Lyon, MATEIS CNRS UMR5510, Villeurbanne, France
}

\begin{abstract}
Porous, strong, and stiff ceramic materials are required for a range of technical applications involving, for instance, liquid or gas flow. Natural materials such as wood can provide useful structural guidelines for the optimal microstructural design, although few processing route are able to turn these guidelines into actual materials. We illustrate here how ice templating of anisotropic particles suspensions can be modified to obtain a honeycomb structure with pores of $30 \mu \mathrm{m}$. The growth of ice crystals in the slurry induces self-assembly of the anisotropic particles, leading to relatively thin walls $(10 \mu \mathrm{m})$. Because large anisotropic particles are difficult to sinter, a glassy phase was introduced to facilitate this densification step and then to further reduce the walls' porosity. Young's modulus and compressive strength were both improved by the addition of a glassy phase by an order of magnitude due to the denser walls. These macroporous materials are more robust and stiff than materials with an equivalent morphology, while offering a simple alternative to the current wood replica processing routes.
\end{abstract}

\section{Introduction}

The mechanical properties of porous cellular materials directly depend on their cell structure. Natural materials such as wood, because their role is to withstand stresses and to mediate water and nutriments transfer, provide guidelines to obtain cellular materials with remarkable stiffness and tailored porosity. The most common cellular structure encountered in load-bearing natural material is the honeycomb because this geometry provides a very high specific elastic modulus ${ }^{1}$. The pores need to be as parallel and straight as possible while the hexagonal shape maximizes the structural stiffness.

The options to obtain cellular ceramic materials with an oriented, anisotropic porosity are limited when the targeted cell size and cell wall thickness are similar to that of wood (typically $30 \mu \mathrm{m}$ cell size and less than $5 \mu \mathrm{m}$ in wall thickness for balsa ${ }^{2}$ ). Wood structures are defined by a delicate balance between the densities of pores (to have a large accessible area), large and straight pores to limit the pressure drop, and cell 
structure and wall thickness to provide structural resistance. Cellular ceramics presenting such characteristics can be useful as supports for liquid or gaseous chemical reaction and more generally as fluid carrier. Usual ceramic foams partly fulfill those requirements, such as a high porosity content and high strength, but their low cell connectivity and high tortuosity may restrain their use for those applications. The presence of straight and oriented pores can greatly increase the permeability of a porous support $^{3,4}$.

Today the most common way to obtain cellular material is extrusion, although technical limitations make it extremely complicated to decrease the pore size below a hundred of micrometers or so. Alternatively, direct replication of the wood structure has already been successfully demonstrated ${ }^{5-7}$. The use of natural wood templates and multiple heat/impregnation treatments can lead to ceramic materials where cellulose is converted into a ceramic phase. Even if the template structure is preserved, which helps retain the mechanical properties, the large number of steps and specificity of the chemistry involved remain important drawbacks ${ }^{8}$. Here we present an alternative and simpler process, based on ice templating, to obtain porous ceramics emulating the structure of wood.

Ice templated porous materials have already demonstrated appealing mechanical properties arising from the three dimensional architecture of the structure imprinted by the ice crystals ${ }^{9}$. The process is based on the phase separation that occurs when a colloidal suspension is frozen, leading to the rejection of the particles by the growing crystals. Because of a strong anisotropy of crystal growth velocities, ice crystals adopt a lamellar morphology. The structure is lamellar and mechanical properties are thus maximized along this direction. The tortuosity of the porous phase in these structures remains low. This process has been successfully used to make unidirectional porous support, with cell size and wall thickness approaching that of natural balsa. Some are based on camphene suspension ${ }^{10,11}$ and the materials obtained present dendritic and tortuous pore morphology. Others, based on jellified water-based suspensions, get close to the structure of the natural model but their strength and stiffness are usually rather low ${ }^{12}$, except for silicon carbide ones ${ }^{13}$.

A $30 \mu \mathrm{m}$ pore diameter can be obtained with a slow cooling rate (around $-1^{\circ} \mathrm{C} / \mathrm{min}$ ), resulting in a low growth velocity ${ }^{14}$. Such conditions nevertheless result in highly tilted pores. Large particles, around a few micrometers, can stabilize the interface and reduce the crystal tilt, resulting in straight and equiaxed crystals at the expense of a greater walls thickness.

In order to obtain a honeycomb-like porous structure with thin ceramic walls, in the present paper we use a combination of an ice shaping compound and anisotropic, platelet shaped particles. The growing ice crystals trigger the self-assembly of the 
platelets ${ }^{15}$. Different mechanisms have been proposed for this alignment, such as shear forces at crystal tip $^{16}$ or anisotropic growth rates ${ }^{17}$. Walls constituted of aligned platelets can be significantly thinner than those composed of spheres because the percolation threshold is inversely proportional to the aspect ratio. Simultaneously, we take advantage of the ice shaping properties of zirconium acetate ${ }^{18,19}$ (ZRA) to obtain hexagonal ice crystals and thus a honeycomb-like porous architecture.

We show that this combination can lead to stiff and strong porous cellular ceramic materials with specific mechanical properties approaching that of wood.

\section{Experimental procedure}

Suspensions were prepared by mixing distilled water with a zirconium acetate (SaintGobain) leading to a concentration of zirconium around $20 \mathrm{~g} / \mathrm{L}$, an organic binder (Poly Ethylene Glycol 20M, 4 wt.\% of dried powder mass, Sigma Aldrich), and the alumina powder (RonaFlair White Sapphire; produced by Antaria ltd, sold by Merck KGaA) at 13 vol.\%. This concentration of zirconium acetate lead to a suspension $\mathrm{pH}$ of 4 , a necessary condition to obtain hexagonal pores ${ }^{18}$. The particles used in the study were platelets with a diameter around $8 \mu \mathrm{m}$ and a thickness of $500 \mathrm{~nm}$. Isotropic alumina particles were also used for comparison (Ceralox SPA05, Ceralox Div., Condea Vista Co., Tucson, AZ). Slurries were ball-milled for $20 \mathrm{~h}$ with alumina milling media. The glassy phase precursors consisted of a colloidal suspension of silica (Nexsil 20K, diameter $20 \mathrm{~nm}$, provide by Nyacol) and calcium nitrate tetrahydrate (Sigma Aldrich). The ratio of each precursor was adjusted to obtain a composition of 75:25 molar ratio of Si:Ca ions and 5 or 10 vol \% were added based on alumina content.

Freezing of the slurries was done by pouring them into a silicone mold (20 $\mathrm{mm}$ diameter, $21 \mathrm{~mm}$ height) placed on a cooled copper plate. The copper plate was cooled by silicone oil at a temperature regulated by a cryothermostat. The silicon oil temperature was regulated so that a cooling rate of $-1^{\circ} \mathrm{C} / \mathrm{min}$ was achieved on the copper plate. Once freezing was completed, the samples were freeze-dried for at least $48 \mathrm{~h}$ in a commercial freeze-dryer (Free Zone 2.5 Plus, Labconco, Kansas City, Missouri, USA) to ensure a complete removal of the ice crystals. The heat treatment comprised a binder removal step, heating from room temperature to $550^{\circ} \mathrm{C}$ at $50^{\circ} \mathrm{C} / \mathrm{h}$ followed by a steady stage of $2 \mathrm{hrs}$. Directly after this organic burnout, the samples were sintered at $1550^{\circ} \mathrm{C}$ for $2 \mathrm{hrs}$ with $300^{\circ} \mathrm{C} / \mathrm{h}$ heating and cooling rates.

Geometrical density measurements were made after the removal of one millimeter of material at the top and the bottom of each sample to avoid surface artifacts. The theoretical bulk density was calculated by a rule of mixture to take into account the addition of the glassy phase. The phase volume fractions for the rule of mixture were calculated accordingly to the amount of precursors introduced in the suspensions. The 
following theoretical densities were used: $3.97 \mathrm{~g} / \mathrm{cm}^{3}$ for alpha alumina, $2.20 \mathrm{~g} / \mathrm{cm}^{3}$ for silica, and $3.3 \mathrm{~g} / \mathrm{cm}^{3}$ for calcia.

Pore diameters and volume were measured by mercury intrusion porosimetry (AutoPore IV 9500, Micromeritics) with applied pressures up to $200 \mathrm{MPa}$. The open porosity of the pore walls was deduced from the volume $\mathrm{V}_{\mathrm{m}}$ of mercury introduced below a $10 \mu \mathrm{m}$ equivalent pore diameter and the volume of the sample $V_{e}$ by the following relation: walls open porosity $=\mathrm{V}_{\mathrm{m}} /\left(\mathrm{V}_{\mathrm{m}}+\mathrm{V}_{\mathrm{e}}\right)$.

Samples (dimensions around $18 \mathrm{~mm}$ in diameter and $18 \mathrm{~mm}$ height) surfaces were first ground to be parallel then the samples were compressed on a testing machine (Shimadzu AGSX), with a crosshead speed of $0.2 \mathrm{~mm} / \mathrm{min}$.

\section{Results and discussion}

The typical microstructure of a sample made by ice templating with alumina platelets and ZRA is shown in figure 1a. The macropores (figure 1c) are approximately hexagonal, with a relatively smooth surface resulting from the local alignment of the platelets. The structure also presents a unique pore connectivity and morphology. The pores are arranged in elongated cells (figure 2). This feature is possibly a consequence of the large size of particles, able to induce tip splitting of the ice crystals ${ }^{20}$. The morphology of these materials is thus unexpectedly similar to that of wood, which presents the same cell structure ${ }^{2,21}$. A sample made of isotropic $0.5 \mu \mathrm{m}$ alumina particles obtained under the same freezing conditions is shown in figure 1b. The material containing isotropic particles presents highly tilted pores (approximately at $45^{\circ}$ of the direction of the temperature gradient) characteristic of the structures obtained at low growth velocities (figure 1b). The mechanical properties of cellular materials strongly depend on the pore volume and properties of the solid phase. Here the particle rich phase is porous because the ice crystals repel the particles until a critical particle concentration is reached ${ }^{22}$. This concentration is typically around the random close packing for monodispersed spherical particles but lower for less isotropic particles. This porosity drastically impacts the mechanical properties ${ }^{16}$ and thus needs to be reduced. For submicromic ceramic particles, the remaining porosity is reduced by a sintering step. In this study, the particles exhibited a $8 \mu \mathrm{m}$ diameter and a $500 \mathrm{~nm}$ thickness, yielding a ceramic phase with lower green and sintered densities. Indeed, the volume of such particles and the low number of particle contacts (due to the high aspect ratio) prevent their rearrangement and coarsening. The Laplace pressure is not sufficient for densification in this case. To improve the mechanical properties, the porosity of the walls must therefore be reduced.

The solution chosen here was to densify by liquid phase sintering. If the glassy phase composition and volume are suitable, its presence can facilitate particle rearrangement. 
The glassy phase must exhibit a good wettability with particles so that the capillary forces induced by the liquid film between adjacent particles are strong enough to bring them closer while the liquid partially fills the gap. A glassy phase composed of silica and calcia in molar ratio 75:25 was chosen. Previous studies ${ }^{23,24}$ reported a good wettability of this compound with alumina, in conjunction with formation of a calcium aluminosilicate phase at the grain boundary. An intergranular phase can further enhance the contact strength between adjacent particles. The use of such a glassy phase allows us to decrease the wall porosity in the sample and thus improve the mechanical properties.

The addition of a glassy phase allows densification of the sample during sintering which does not occur in samples without sintering additives (figure 3a). However, the macropore size is relatively independent of the amount of glassy phase (figure $3 \mathrm{c}$ ). The shrinkage is therefore due to densification of the ceramic walls. The pore network, assessed by mercury intrusion, is characterized by two different peaks: one corresponding to the intergranular porosity (figure $3 \mathrm{~b}$ ) and another for macroporosity (figure 3c). The intergranular porosity is significantly decreased by the addition of glassy phase (figure 3b). By comparing the amount of the two porosity types with the material volume, the average wall porosity can be calculated as a function of glassy phase content.

The walls composed only of platelets present a high pore content (60\%). The only change in the structure after sintering consists of necks created by diffusion at the few contact points between adjacent platelets. The porosity is thus the same as in the freeze-dried green body. The wall density is lower than the one obtained with spherical particles ${ }^{25}$ due to the smaller packing ability of high aspect ratio particles. Because of this high porosity, the compressive strength is relatively low. The addition of 5 and 10 vol. $\%$ of glassy phase reduces the wall open porosity from $60 \%$ to $18 \%$ and $4 \%$ (figure 4a), respectively, which in turn increases the compressive strengths by a factor of 4 and 7, respectively (figure 4b). The Young's modulus increase by a factor of 4 (10 vol.\% of glassy phase, figure 4c). The tilted pore structure obtained with spherical particles results in low compressive strength (2.1 MPa) and Young's modulus (75 MPa), despite a density slightly higher than the samples with glassy phase $\left(0.78 \mathrm{~g} / \mathrm{cm}^{3}\right.$ and $0.73 \mathrm{~g} / \mathrm{cm}^{3}$ respectively). The presence of a glassy phase and rearrangement are illustrated in figure $4 \mathrm{~d}$ and figure 4e. Clearly, the addition of glassy phase in the structure greatly improves the mechanical properties by allowing a particle rearrangement, which results in a reduction of the porosity in the walls after sintering.

The mechanical response of the material is drastically modified by the addition of the glassy phase. Not only does the glassy phase fraction increase the compressive strength and Young's modulus, but it also affects the fracture behavior. Typical stress-strain curves for the three different types of materials are shown in figure 5 . The behavior of 
the sample containing 5 vol. $\%$ of glassy phase is non-linear after a stress of $8 \mathrm{MPa}$. Observations of the fractured samples revealed localized damage of the sample in contact with the dies. The two other compositions are characterized by brittle facture behavior, arising from unresolved tensile stresses due to the friction on the dies. Along with the increase of mechanical properties, the amount of glassy phase modifies the fracture behavior of the samples. By decreasing the porosity in the walls, a compromise is reached when the porosity is low enough to obtain interesting macroscopic properties and at the same time high enough to promote gradual crushing instead of a catastrophic failure (here at 5 vol.\% of glassy phase). The failure of the sample under load is progressive, a behavior similar to that observed in ceramic foams. When the porosity in the wall becomes too low (10 vol.\% of glassy phase), the usual failure mechanisms are observed with longitudinal crack propagation throughout the sample.

The strengths of the porous structures obtained here are plotted in figure 6 , along with similar structures and macroporous foams made of alumina. The strengths of our materials are closed to the maximum of the one obtained with foams with the same level of porosity. The two materials close to that are a camphene-based material ${ }^{11}$ the alumina wood replica ${ }^{7}$, which are made by complex and multi steps processes. The specific Young's moduli of our materials and the ones with similar microstructure are presented in table I. The structures reported by Hunger et al. ${ }^{16}$ present approximately the same pore sizes and wall thicknesses but are comprise polymer (gelatin and chitosan) and alumina platelets. The presence of a polymeric phase and a lamellar pore structure decreases the stiffness and resistance of the unit cell. Rambo et al. ${ }^{7}$ use an Al-vapor deposition process to transform natural wood into $-\mathrm{Al}_{2} \mathrm{O}_{3}$ while retaining the wood microstructure. However, the specific stiffness is clearly below natural wood $(0.11$ $\mathrm{MPa} /\left(\mathrm{g} / \mathrm{cm}^{3}\right)$ compared to $\left.10 \mathrm{MPa} /\left(\mathrm{g} / \mathrm{cm}^{3}\right)\right)$ due to the presence of defects in the wall that, according to the authors, arise from the deposition. The specimen showed in this study presented higher characteristics because of a stiffer and defect-free unit cell material. However, the properties of these materials are still not quite at the level of natural balsa in terms of specific stiffness $\left(0.64 \mathrm{MPa} /\left(\mathrm{g} / \mathrm{cm}^{3}\right)\right.$, see table I). Another wood replica was obtained by pyrolysis ${ }^{5}$, leading to a carbon-based structure with specific properties close to that of the template $\left(4 \mathrm{MPa} /\left(\mathrm{g} / \mathrm{cm}^{3}\right)\right)$. The walls probably retained the structure of wood at a smaller scale, such as the crystallographic alignment of cellulose fibers, which leads to an increased stiffness.

The materials obtained share certain structural features with wood, such as hexagonal cells of similar dimensions (around $30 \mu \mathrm{m}$ ) and relatively thin cell walls. These bioinspired materials exhibit interesting mechanical properties compared to other similar structures reported in the literature but also compared to other alumina foam made by various techniques. However, they are still short of the value of wood or its carbon replica by an order of magnitude in stiffness. The ice templated materials obtained here do not mimic all of the complex wood architecture, and in particular the 
internal structure of the cell's wall. The benefit of the trade-off is the relative simplicity and versatility of the processing approach presented here, compared to the wood replica techniques.

\section{Conclusions}

Cellular ceramics have been synthesized with structural guidelines derived from wood. To reproduce wood-like features, the ice templating process has been used to obtain large and straight pores with self-assembled platelets during freezing. Because the honeycomb cell structure is known to maximize the specific Young's modulus, zirconium acetate addition was used to promote such a structure. The harmful effect of platelets on densification of the walls formed after ice templating has been countered by the addition of a glassy phase. The resulting specific properties are relatively close to that of wood in terms of compressive strength but still an order of magnitude short in stiffness. Indeed, the macrostructure of wood have been successfully mirrored here, but only at the length scale of macropores. Further work to control the microstructure at a finer scale is required.

\section{References}

1. Gibson, L. J. \& Ashby, M. F. Cellular Solids: Structure and Properties. 532 (Cambridge Univ Press, 1999).

2. Da Silva, A. \& Kyriakides, S. Compressive response and failure of balsa wood. Int. J. Solids Struct. 44, 8685-8717 (2007).

3. Scheffler, M. \& Colombo, P. Cellular Ceramics: Structure, Manufacturing, Properties and Applications. 670 (Wiley-VCH (Weinheim), 2005).

4. Isobe, T., Kameshima, Y., Nakajima, A., Okada, K. \& Hotta, Y. Gas permeability and mechanical properties of porous alumina ceramics with unidirectionally aligned pores. J. Eur. Ceram. Soc. 27, 53-59 (2007).

5. Greil, P., Lifka, T. \& Kaindl, A. Biomorphic Cellular Silicon Carbide Ceramics from Wood: II. Mechanical Properties. J. Eur. Ceram. Soc. 18, 1975-1983 (1998).

6. Vogli, E., Sieber, H. \& Greil, P. Biomorphic SiC-ceramic prepared by Si-vapor phase infiltration of wood. J. Eur. Ceram. Soc. 22, 2663-2668 (2002).

7. Rambo, C. R. et al. Microcellular Al 2 O 3 Ceramics from Wood for Filter Applications. J. Am. Ceram. Soc. 91, 852-859 (2008).

8. Studart, A. R., Gonzenbach, U. T., Tervoort, E. \& Gauckler, L. J. Processing Routes to Macroporous Ceramics: A Review. J. Am. Ceram. Soc. 89, 1771-1789 (2006).

9. Deville, S. Ice templating, freeze casting: Beyond materials processing. J. Mater. Res. 28, 1-18 (2013). 
10. Hong, C., Zhang, X., Han, J., Du, J. \& Han, W. Ultra-high-porosity zirconia ceramics fabricated by novel room-temperature freeze-casting. Scr. Mater. 60, 563-566 (2009).

11. Moon, Y.-W., Shin, K.-H., Koh, Y.-H., Choi, W.-Y. \& Kim, H.-E. Porous alumina ceramics with highly aligned pores by heat-treating extruded alumina/camphene body at temperature near its solidification point. J. Eur. Ceram. Soc. 32, 1029-1034 (2012).

12. Fukushima, M., Nakata, M. \& Yoshizawa, Y. Fabrication and properties of ultra highly porous cordierite with oriented micrometer-sized cylindrical pores by gelation and freezing method. J. Ceram. Soc. Japan 116, 1322-1325 (2008).

13. Fukushima, M., Nakata, M., Zhou, Y., Ohji, T. \& Yoshizawa, Y. Fabrication and properties of ultra highly porous silicon carbide by the gelation-freezing method. J. Eur. Ceram. Soc. 30, 2889-2896 (2010).

14. Deville, S., Saiz, E., Nalla, R. K. \& Tomsia, A. P. Freezing as a path to build complex composites. Science (80-. ). 311, 515-8 (2006).

15. Qiu, L., Liu, J. Z., Chang, S. L. Y., Wu, Y. \& Li, D. Biomimetic superelastic graphene-based cellular monoliths. Nat. Commun. 3, 1241 (2012).

16. Hunger, P. M., Donius, A. E. \& Wegst, U. G. K. K. Platelets self-assemble into porous nacre during freeze casting. J. Mech. Behav. Biomed. Mater. 19, 87-93 (2013).

17. Lee, J. \& Deng, Y. The morphology and mechanical properties of layer structured cellulose microfibril foams from ice-templating method. Biomacromolecules 7, 6034 (2011).

18. Deville, S. et al. Ice shaping properties, similar to that of antifreeze proteins, of a zirconium acetate complex. PLoS One 6, e26474 (2011).

19. Deville, S., Viazzi, C. \& Guizard, C. Ice-structuring mechanism for zirconium acetate. Langmuir 28, 14892-8 (2012).

20. Sekhar, J. A. \& Trivedi, R. Solidification microstructure evolution in the presence of inert particles. Mater. Sci. Eng. A 147, 9-21 (1991).

21. Vural, M. \& Ravichandran, G. Microstructural aspects and modeling of failure in naturally occurring porous composites. Mech. Mater. 35, 523-536 (2003).

22. Shanti, N. O., Araki, K. \& Halloran, J. W. Particle Redistribution During Dendritic Solidification of Particle Suspensions. J. Am. Ceram. Soc. 89, 24442447 (2006).

23. Seabaugh, M. M., Kerscht, I. H. \& Messing, G. L. Texture Development by Templated Grain Growth in Liquid Phase Sintered alpha-Alumina. J. Am. Ceram. Soc. 80, 1181-1188 (1997).

24. Pavlacka, R. J. \& Messing, G. L. Processing and mechanical response of highly textured Al2O3. J. Eur. Ceram. Soc. 30, 2917-2925 (2010). 
25. Peppin, S. S. L., Elliott, J. a. W. \& Worster, M. G. Solidification of colloidal suspensions. J. Fluid Mech. 554, 147 (2006).

26. Gonzenbach, U. T., Studart, A. R., Tervoort, E. \& Gauckler, L. J. Macroporous Ceramics from Particle-Stabilized Wet Foams. J. Am. Ceram. Soc. 90, 16-22 (2007).

27. Hunger, P. M., Donius, A. E. \& Wegst, U. G. K. Structure-property-processing correlations in freeze-cast composite scaffolds. Acta Biomater. 9, 6338-48 (2013).

28. Yoon, H.-J. et al. Macroporous Alumina Ceramics with Aligned Microporous Walls by Unidirectionally Freezing Foamed Aqueous Ceramic Suspensions. J. Am. Ceram. Soc. 1582, 2009-2011 (2010).

29. Han, J., Hong, C., Zhang, X., Du, J. \& Zhang, W. Highly porous ZrO2 ceramics fabricated by a camphene-based freeze-casting route: Microstructure and properties. J. Eur. Ceram. Soc. 30, 53-60 (2010). 
Table I: Comparison of specific Young's modulus for different materials with similar architecture

\begin{tabular}{|l|l|}
\hline Material and reference & $\begin{array}{l}\text { Specific Young's } \\
(\mathrm{GPa} / \mathrm{g} / \mathrm{cm} 3))\end{array}$ \\
\hline Alumina platelets (this study) & 0.64 \\
\hline Alumina spheres (this study) & 0.10 \\
\hline Alumina platelets/chitosan ${ }^{16}$ & 0.13 \\
\hline Alumina spheres/chitosan ${ }^{27}$ & 0.03 \\
\hline Alumina wood replica ${ }^{7}$ & 0.11 \\
\hline Zirconia ice-templated with camphene ${ }^{29}$ & 0.53 \\
\hline Carbon wood replica $^{5}$ & 4.08 \\
\hline Balsa wood $^{2}$ (transverse / longitudinal) $^{2}$ & $1 / 10$ \\
\hline
\end{tabular}



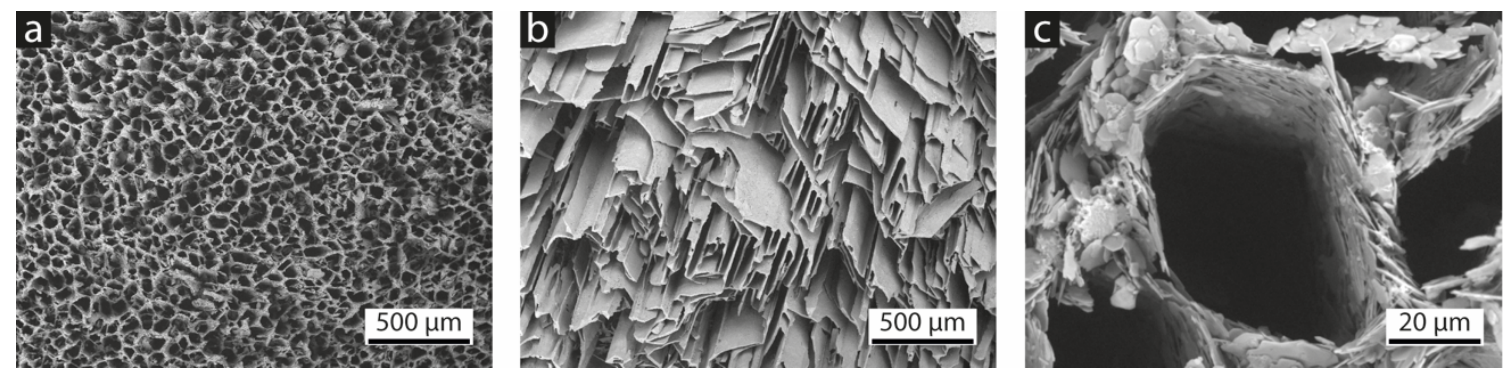

Figure 1: SEM micrographs of porous alumina structures obtained under the same conditions with (a) large alumina platelets and (b) small isotropic particles. Crosssection perpendicular to the temperature gradient. (c) Closer view of a macropore.

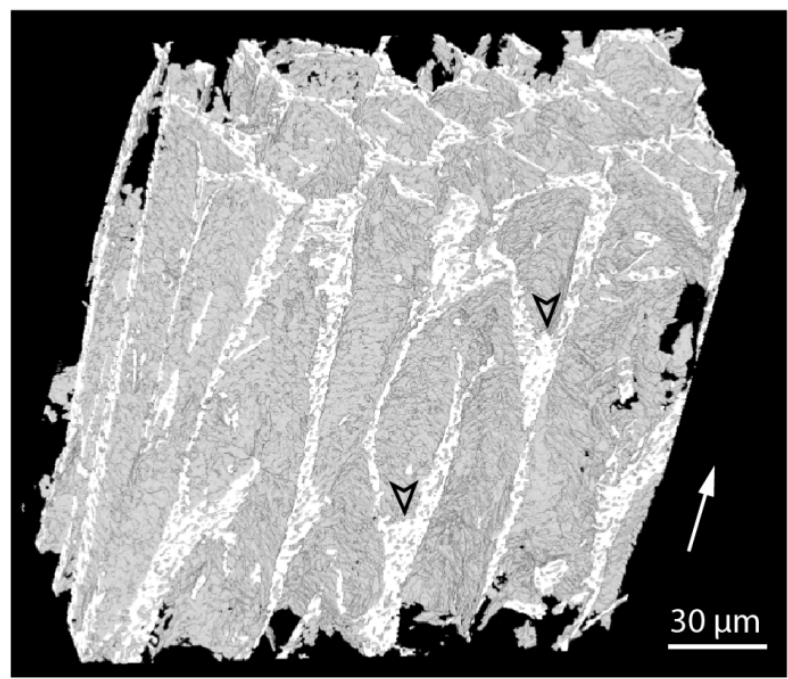

Figure 2: 3D structure of the structure obtained by tomography containing alumina platelets and 10 vol.\% of glassy phase. The arrows indicate the beginning of a new cell. The white arrow indicates the solidification direction. 

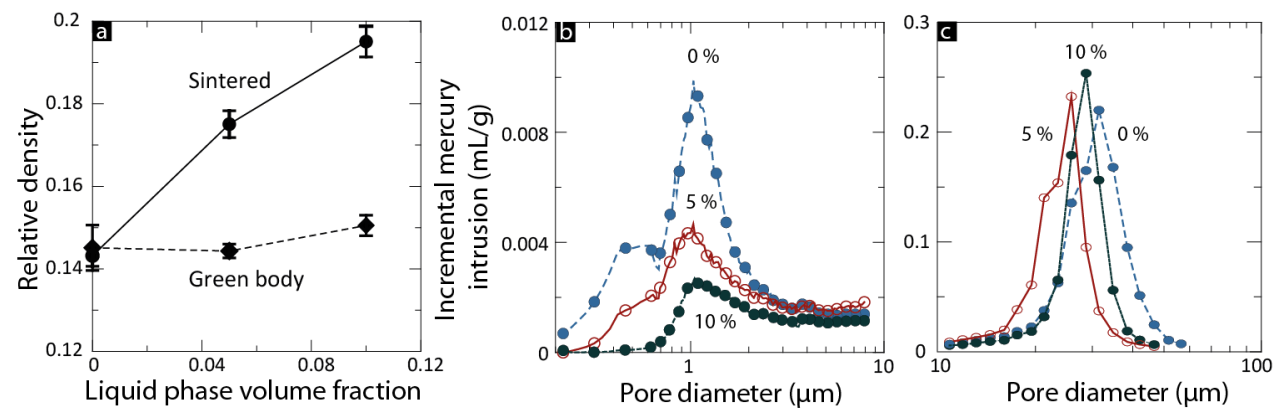

Figure 3: Evolution of (a) the relative density, (b) the intergranular and (c) the macroporosity of the cellular material with alumina platelets vs. the amount of glassy phase.
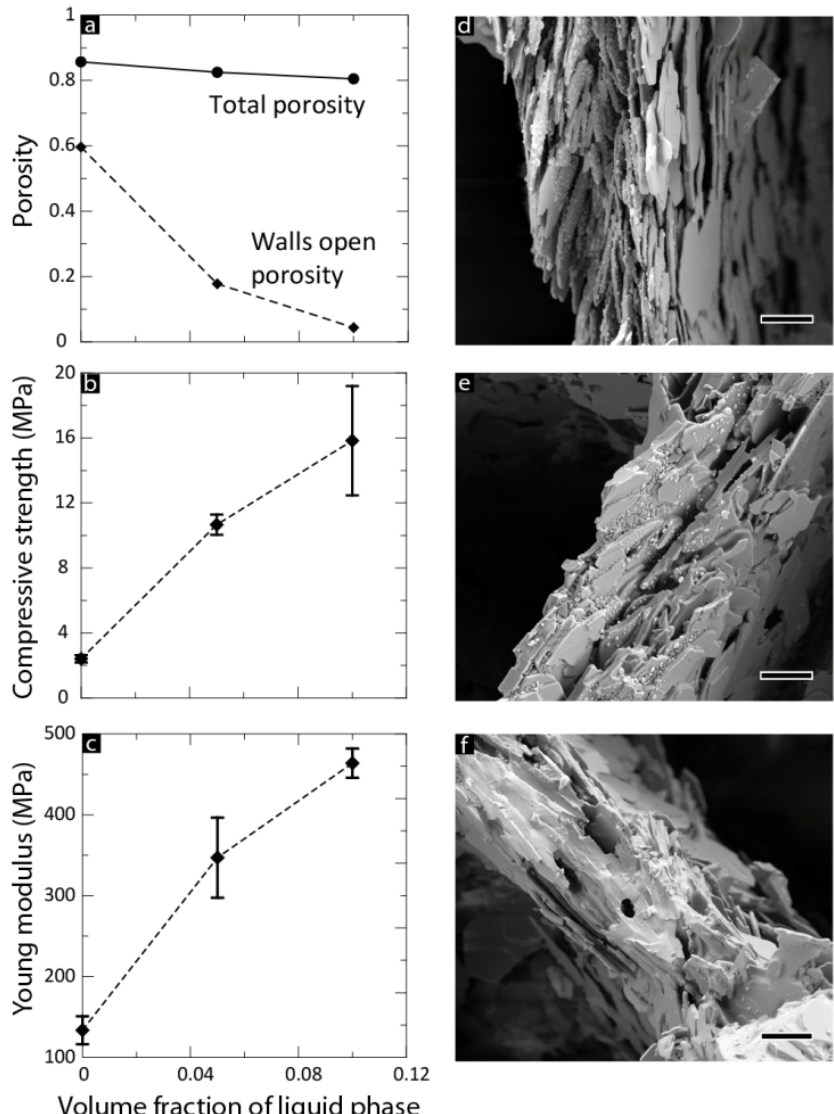

Figure 4: Influence of glassy phase content on the structural and mechanical properties of the cellular material containing alumina platelets. SEM pictures of structure with (d) 0 vol.\%, (e) 5 vol.\%, and (f) 10 vol.\% of glassy phase. Scale bars: $5 \mu \mathrm{m}$. 


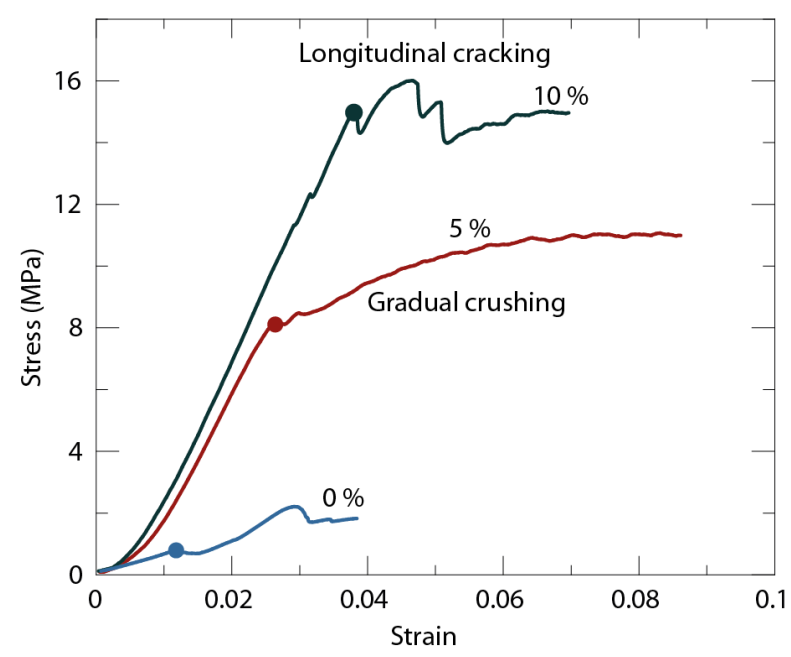

Figure 5: Typical stress-strain curves obtained under compression as a function of the glassy phase content. Dots represent the first recorded crack propagation.

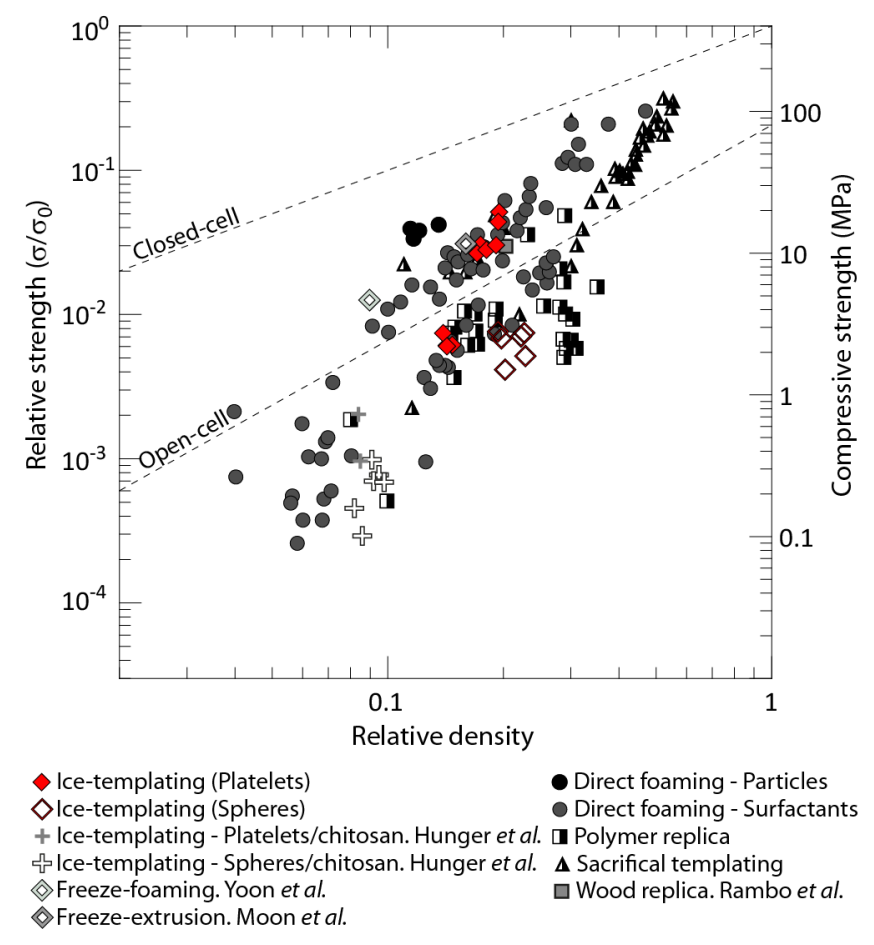

Figure 6: Compressive strength of our ice templated alumina structures compared to macroporous alumina obtained by various techniques: direct foaming, polymer replica, sacrificial templating ${ }^{26}$, wood replica ${ }^{7}$, ice templating of composites ${ }^{16,27}$, freezing/foaming ${ }^{28}$ and freezing/extrusion ${ }^{11}$. Reproduced from Gonzenbach et al. ${ }^{26}$ 